\title{
TAUVEX AND THE NATURE OF THE COSMOLOGICAL UV BACKGROUND
}

\author{
NOAH BROSCH \\ The School of Physics and Astronomy, Raymond and Beverly \\ Sackler Faculty of Exact Sciences, Tel Aviv University, Tel \\ Aviv 69978, Israel
}

TAUVEX is a three-telescope array intended to image wide sky areas in the UV. It is being constructed in Israel for flying on-board the Spectrum X- $\gamma$ (SRG) international high-energy observatory. SRG will be orbited by Russia in early 1996 for a three-year + mission. TAUVEX will operate in parallel with X-ray imaging telescopes on board SRG to provide timeresolved photometry and deep UV imaging.

Observations in the UV region longward of Lyman $\alpha$ up to the atmospheric limit at $\sim 3000 \AA$ take advantage of reduced sky background because of a fortuitous combination of zodiacal light decreasing shortward of $\sim 3000 \AA$ and other backgrounds remaining low up to near the geocoronal Lyman $\alpha$. In this spectral region it is therefore possible to observe faint astronomical sources with high signal-to-noise ratio, even with a modest telescope.

The present design of TAUVEX includes three co-aligned $20 \mathrm{~cm}$ diameter telescopes in a linear array on the same mounting surface. Each telescope images a field of $0^{\circ} .9$ with $10^{\prime \prime}$ resolution onto photon-counting positionsensitive detectors with wedge-and-strip anodes, produced by DEP of Roden, Holland. The payload is designed and assembled by El-Op ElectroOptics Industries, Ltd., of Rehovot, the top electro-optical manufacturer of Israel, under close supervision of Tel Aviv University astronomers.

The TAUVEX telescopes are equipped with a set of six filters, which sample the spectral segment $1400-2800 \AA$. Almost all sub-assemblies and parts are at least doubly-redundant, and in most cases the redundancy is invoked automatically. Significant image processing is done on-board, to reduce the volume of the downlinked data.

The combination of long observing periods of SRG (three days out of every four), a high orbit (200,000 $\mathrm{km}$ apogee) with low radiation and solar 
scattered background, and long staring sessions to every target (typically 4-6 hours), implies that TAUVEX will be able to detect and measure starlike objects of $20.5 \mathrm{mag}$ with a $\mathrm{S} / \mathrm{N}$ of 10 . This corresponds to $\mathrm{V} \simeq 22.5 \mathrm{mag}$ QSOs, given typical [UV-V] colors of QSOs, and at least 10 such objects are expected in every TAUVEX field-of-view. During the guaranteed SRG mission, at least 50,000 QSOs will be observed, if the targets will be different and at high galactic latitude. This is an order of magnitude more QSOs than cataloged now.

Diffuse objects, such as nearby large galaxies, will be measured to a surface brightness of about $20 \mathrm{UV}$ mag/square arcsec for each resolution element. Combining the information for larger areas will reach much fainter surface magnitudes. The UV sky background has been reviewed by Bowyer (1991) and Henry (1991). The background is correlated with the Galactic HI column density (Bowyer 1990), and the lowest acceptable value is about 100 photons $/ \mathrm{sec} / \mathrm{cm}^{2} / \AA /$ steradian, as derived from the extrapolation to zero HI column density.

The main limitation of TAUVEX in detecting the diffuse UV background is straylight; this becomes problematic for very faint values of brightness whenever the Sun is in the forward direction of SRG. In cases whenever straylight will not be a problem the detection will be limited by photon statistics, as the TAUVEX detectors are virtually noiseless. In a typical SRG pointing of six hours, using the broad-band filter with HPBW $\simeq 1000 \AA$, TAUVEX will detect the lowest UV background value quoted above with a $\mathrm{S} / \mathrm{N} \geq 5$ in each 10 " resolution element. Other filters may be used, with a corresponding reduction of $\mathrm{S} / \mathrm{N}$, or this may be boosted by area binning, yielding the wavelength distribution of the UV sky background.

The results have important implications in understanding the nature of the UV background and its astrophysical sources. For example, is it indeed only the summed UV emission from galaxies that produces the extragalactic component? What is the contribution of resolved galaxies at intermediate redshifts, and how do Butcher-Oemler galaxies fit in this picture?

\section{References}

Bowyer, S. (1991) The cosmic far ultraviolet background, Ann. Rev. Astr. Astrophys. Vol. no. 29, pp. 59-88.

Bowyer, S. (1990) The galactic far ultraviolet background, in The Galactic and Extragalactic Background Radiation (S. Bowyer and Ch. Leinert, eds.), Kluwer Academic Press, pp. 171-18.

Henry, R.C. (1991) Ultraviolet background radiation, Ann. Rev. Astr. Astrophys. Vol. no. 29, pp. 89-127. 